\title{
THE FINITE DIFFERENCE METHOD ON ADAPTIVE MESH FOR SINGULARLY PERTURBED NONLINEAR 1D REACTION DIFFUSION BOUNDARY VALUE PROBLEMS
}

\author{
Hakkı Duru, Baransel Güneş \\ Department of Mathematics, Van Yuzuncu Yil University \\ Van, Turkey \\ hakkiduru@gmail.com, baranselgunes23@gmail.com
}

Received: 10 June 2020; Accepted: 12 November 2020

\begin{abstract}
In this paper, we study singularly perturbed nonlinear reaction-diffusion equations. The asymptotic behavior of the solution is examined. The difference scheme which is accomplished by the method of integral identities with using of interpolation quadrature rules with weight functions and remainder term integral form is established on adaptive mesh. Uniform convergence and stability of the difference method are discussed in the discrete maximum norm. The discrete scheme shows that orders of convergent rates are close to 2. An algorithm is presented, and some problems are solved to validate the theoretical results.
\end{abstract}

MSC 2010: 65L10, 65L11, 65L12

Keywords: boundary value problem, singularly perturbed problem, finite difference method

\section{Introduction}

This study is concerned with following the singularly perturbed boundary value problem in which a second-order derivative is multiplied by a small positive parameter $\varepsilon^{2}$

$$
\begin{gathered}
-\varepsilon^{2} u^{\prime \prime}+a(x) u(x)=f(x, u) ; \quad 0 \leq x \leq l, \\
u(0)=\kappa_{0}, u(l)=\kappa_{1},
\end{gathered}
$$

where $0<\varepsilon \ll 1$ is the perturbation parameter, $\kappa_{0}$ and $\kappa_{1}$ are given constants. $a(x)$ and $f(x, u)((x, u) \in[0, l] \times \mathbb{R})$ are given sufficiently smooth functions. Moreover

$$
\frac{\partial f}{\partial u} \geq p>0 \text { in }[0, l] \times \mathbb{R} .
$$

Reaction-diffusion equations are typical mathematical models in many areas of science and engineering. Nuclear reactivity, population dynamics, biological 
systems, a control theory, fluid dynamics, chemical processes and finance are among these [1-4]. For example, travelling waves of chemical concentration were dealt with by fractional reaction-diffusion equations [5]. Furthermore, the following equation has been presented for models of the spread of invasive species

$$
\frac{\partial u(x, t)}{\partial t}=D \frac{\partial^{2} u(x, t)}{\partial^{2} t}+f(u(x, t))
$$

where $u(x, t)$ is the population density and $x \in \mathbb{R}[4]$. Another sample has been introduced for blow-up profiles

$$
\begin{gathered}
u_{t}=\Delta u+e^{u}, x \in \Omega, \mathrm{t}>0, \\
u(x, 0)=u_{0}(x) \geq 0,
\end{gathered}
$$

where $|x|<R$ and $u_{0}$ is non-negative [6].

Different forms of reaction-diffusion equations have been located in literature. Some authors have taken interest in numerical solutions of these equations. Therefore, there are many different types of numerical approaches that have been presented. The implicit Runge-Kutta method was used for time-dependent reactiondiffusion equations [7]. The explicit Euler discretized is considered for nonlinear form [3]. The implicit Galerkin-Legendre spectral method is applied to the two dimensional fractional form [2]. We see the singularly perturbed form of these equations in recent studies. Standard and stabilised finite difference methods are carried out on a Shishkin mesh for semilinear singularly perturbed reactiondiffusion boundary value problems [8]. By using the method of two-steps expansions, nonlocal equations are studied [9]. Anisotropic nonconforming finite element methods are considered with the interpolation postprocessing technique [10]. Considering the Richardson technique, an improved scheme is constructed [11]. The Richardson extrapolation method with the fourth-order stable central difference method were carried out for the self-adjoint type of these equations [12]. Using the compact finite difference method, error bounds are obtained for 1D forms [13]. The standard implicit Euler method and the HODIE compact fourth order finite difference scheme were used [14]. Sobolev gradient methods were used with Dirichlet boundary conditions [15]. By taking into account the finite element method, sign-changing solutions are obtained [16]. The Hybrid finite difference scheme and Numerov scheme are established on a Shishkin type mesh [17]. By carrying out boundary layer analysis, numerical solutions are obtained in a quasi-uniform mesh [18]. The finite difference scheme is constructed for one and two dimensional types [19]. By using asymptotic behavior of the solution, a domain decomposition method is analyzed into three overlapping subdomains [20]. Besides, for delay form of these equations, a fitted finite difference scheme is constructed by using the Numerov's method [1] and a difference scheme is established on a piecewise equadistant mesh [21]. 
Singularly perturbed problems have boundary layers in which the solution changes quickly. In such problems, when $\varepsilon \rightarrow 0$, the boundary layer is getting thinner. Algorithms which are established by traditional numerical techniques are unstable. That's why we need the reliable numerical methods.

Our goal in this paper is to present the robust numerical method for singularly perturbed reaction-diffusion equations on adaptive mesh.

The draft of this research is as follows: In section 2, the properties of the solution for (1)-(2) are handled. In section 3, the difference scheme is constructed on adaptive mesh. In section 4, error approximations are obtained with respect to Bakhvalov mesh transition points. In section 5, the theoretical results are tested on some numerical examples.

\section{Continuous problem}

In this section, we give the asymptotic behavior of the solution and its derivatives, which is required in analysis of the numerical method. For the (1)-(2) nonlinear problem, using the Taylor expansion, we can write

$$
f(x, u)=f(x, 0)+\frac{\partial f(x, \tilde{u})}{\partial u} u,
$$

where $\tilde{u}=\gamma u, 0<\gamma<1$. Also we get $F(x)=f(x, 0)$ and $b(x)=\frac{\partial f(x, \tilde{u})}{\partial u}$. Then

$$
-\varepsilon^{2} u^{\prime \prime}+[a(x)-b(x)] u=F(x) .
$$

If we get $A(x)=a(x)-b(x)$, we obtain

$$
-\varepsilon^{2} u^{\prime \prime}+A(x) u=F(x)
$$

where

$$
\begin{gathered}
A(x)=a(x)-\frac{\partial f(x, \tilde{u})}{\partial u} \geq \alpha>0, \\
F(x)=f(x, 0) \geq 0 .
\end{gathered}
$$

We give the following lemmas for the properties of the solution.

Lemma 1. [22] Let $v(x)$ be a function and satisfy the following conditions:

$$
L v=F(x) \geq 0,
$$

$\kappa_{0} \geq 0, \kappa_{1} \geq 0$. Then $v(x) \geq 0$.

Lemma 2. [22] For a $v(x) \in C[0, l] \cap C^{2}(0, l)$ function, the following estimate is held:

$$
|v(x)| \leq|v(0)|+|v(l)|+\alpha^{-1} \max _{0 \leq s \leq l}|L v(s)|, 0 \leq x \leq l,
$$


Lemma 3. For the solution of the (1)-(2) nonlinear problem, the following estimations are true:

$$
\begin{gathered}
|u(x)| \leq C, 0<x<l ; A(x), F(x) \in \mathrm{C}[0, l], \\
\left|u^{\prime}(x)\right| \leq C\left\{1+\frac{1}{\varepsilon}\left(e^{\frac{-\sqrt{\alpha} x}{\varepsilon}}+e^{\frac{-\sqrt{\alpha}(l-x)}{\varepsilon}}\right)\right\}, 0<x<l, A(x), F(x) \in \mathrm{C}[0, l],
\end{gathered}
$$

Proof. The proof of the lemma is done in a similar manner [22].

\section{Discretization}

In this section, we proceed to construct the difference scheme. Let $\varpi_{N}$ be any non-uniform mesh on $[0, l]$.

$$
\varpi_{N}=\left\{0=x_{1}<x_{2}<\ldots<x_{N-1}<x_{N}=l\right\},
$$

and

$$
\varpi_{N}=\omega_{N} \cup\left\{x_{1}=0, x_{N}=l\right\} .
$$

Before constructing the difference scheme, we define some notation for the mesh functions. For any mesh function we defined on $\varpi_{N}$ :

$$
v_{i}=v\left(x_{i}\right), v_{x, i}=\frac{v_{i+1}-v_{i}}{h_{i+1}}, v_{\hat{x}, i}=\frac{v_{i}-v_{i-1}}{h_{i}}, h_{i}=x_{i}-x_{i-1},
$$

$v_{\bar{x} x, i}=\frac{1}{\hbar_{i}}\left(v_{x, i}-v_{\hat{x}, i}\right)$ with $\hbar_{i}=\frac{1}{2}\left(h_{i}+h_{i+1}\right)$. Node points $x_{i}$ are specified as

$$
x_{i}=\left\{\begin{array}{c}
-\alpha^{-1} \varepsilon \ln \left(1-(1-\varepsilon) \frac{4 i}{N}\right), \quad i=0,1, \ldots, \frac{N}{4}, \quad x_{i} \in\left[0, \sigma_{1}\right], \quad \sigma_{1}<\frac{l}{4} \\
-\alpha^{-1} \varepsilon \ln \left(1-\left(1-e^{-\frac{\alpha l}{4 \varepsilon}}\right) \frac{4 i}{N}\right), \quad i=0,1, \ldots, \frac{N}{4}, \quad x_{i} \in\left[0, \sigma_{1}\right], \quad \sigma_{1}=\frac{l}{4} \\
\sigma_{1}+\left(i-\frac{N}{4}\right) h^{(1)}, i=\frac{N}{4}+1, \ldots, \frac{3 N}{4}, \quad x_{i} \in\left[\sigma_{1}, \sigma_{2}\right], \quad h^{(1)}=\frac{2\left(\sigma_{2}-\sigma_{1}\right)}{N} ; \\
\sigma_{2}-\alpha^{-1} \varepsilon \ln \left(1-(1-\varepsilon) \frac{4\left(i-\frac{3 N}{4}\right)}{N}\right), i=\frac{3 N}{4}+1, \ldots, N, x_{i} \in\left[\sigma_{2}, l\right] ; \\
\sigma_{2}-\alpha^{-1} \varepsilon \ln \left(1-\left(1-e^{-\frac{\alpha l}{4 \varepsilon}}\right) \frac{4\left(i-\frac{3 N}{4}\right)}{N}\right), i=\frac{3 N}{4}+1, \ldots, N, x_{i} \in\left[\sigma_{2}, l\right], \sigma_{2}=\frac{3 l}{4}
\end{array}\right.
$$

To generate the difference method, we begin following integral identity [23]

$$
\hbar_{i}^{-1} \int_{x_{i-1}}^{x_{i+1}}\left(-\varepsilon^{2} u^{\prime \prime}+a(x) u(x)\right) \varphi_{i} d x=\hbar_{i}^{-1} \int_{x_{i-1}}^{x_{i+1}} f(x, u) \varphi_{i} d x
$$


where

Author's corrections

21.10.2022:

It should be: 2

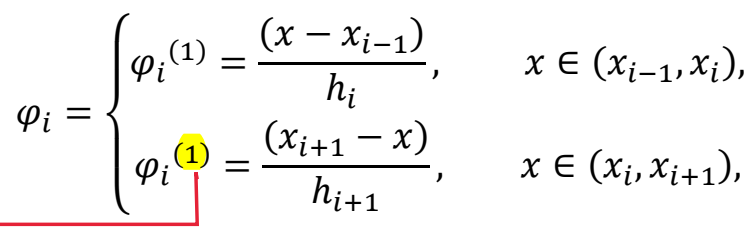

and

$$
\hbar_{i}^{-1} \int_{x_{i-1}}^{x_{i+1}} \varphi_{i} d x=\hbar_{i}^{-1}\left(\frac{h_{i}}{2}+\frac{h_{i+1}}{2}\right)=1
$$

By using interpolating quadrature rules in [24] for each term of (8), we obtain the following difference problem

$$
\begin{gathered}
-\varepsilon^{2} u_{\hat{x} x, i}+a_{i} u_{i}=f\left(x_{i}, u_{i}\right)+R_{i}, i=1, \ldots, N-1, \\
u_{0}=\kappa_{0}, u_{N}=\kappa_{1},
\end{gathered}
$$

where

$$
\begin{gathered}
R_{i}=R_{i}^{(1)}+R_{i}^{(2)}, \\
R_{i}^{(1)}=\hbar_{i}{ }^{-1} \int_{x_{i-1}}^{x_{i+1}} d x \varphi_{i} \int_{x_{i-1}}^{x_{i+1}} \frac{d^{2} u(\xi)}{d \xi^{2}} T_{0}(x-\xi) d \xi \\
R_{i}^{(2)}=-\hbar_{i}^{-1} \int_{x_{i-1}}^{x_{i+1}}\left[a(x)-a\left(x_{i}\right)\right] u(x) \varphi_{i} d x \\
+\hbar_{i}{ }^{-1}\left\{\int_{x_{i-1}}^{x_{i+1}}\left[f(x, u)-f\left(x_{i}, u\right)\right] \varphi_{i} d x+\int_{x_{i-1}}^{x_{i+1}}\left[f\left(x_{i}, u\right)-f\left(x_{i}, u_{i}\right)\right] \varphi_{i} d x\right\} .
\end{gathered}
$$

Hence, we can write the difference problem for the approximate solution $y$

$$
\begin{gathered}
-\varepsilon^{2} y_{\hat{x} x, i}+a_{i} y_{i}=f\left(x_{i}, y_{i}\right), \quad i=1, \ldots, N-1, \\
y_{0}=y_{N}=0 .
\end{gathered}
$$

\section{Error estimates}

To investigate the uniform convergence of the difference method, let $u_{i}$ be the solution (9)-(10) and $y_{i}$ be the solution of (12)-(13). The approximate error $z_{i}=y_{i}-u_{i}, x_{i} \in \omega_{N}$ is the solution of following problem

$$
\begin{gathered}
\varepsilon^{2} z_{\hat{x} x, i}-a_{i} z_{i}=f\left(x_{i}, y_{i}\right)-f\left(x_{i}, u_{i}\right), \quad i=1, \ldots, N-1, \\
z_{0}=z_{N}=0 .
\end{gathered}
$$


By modifying $f\left(x_{i}, y_{i}\right)-f\left(x_{i}, u_{i}\right)$, we can write

$$
l z_{i}=-\varepsilon^{2} z_{\hat{x} x, i}+A_{i} z_{i}=R_{i}, i=1, N-1, z_{0}=z_{N}=0,
$$

where $A_{i}$ is denoted in (3) and $R_{i}$ is given by (11).

Theorem 1. The solution of the difference problem (12)-(13) is uniformly convergent to the solution of the problem (1)-(2) with respect to $\varepsilon$ in $\bar{\omega}_{N}$ under condition $A(x) \in \mathrm{C}^{1}[0, l]$. The following estimate is held

$$
\|y-u\|_{C\left(\bar{\omega}_{N}\right)} \leq \mathrm{C} h_{i}
$$

Proof. For the difference operator $l v_{i}$, if $l v_{i} \geq 0(i=1,2, \ldots, N-1), v_{0} \geq 0$, $v_{N} \geq 0$, we can show $v_{i} \geq 0, i=0,1, \ldots, N$ from the maximum principle. If Lemma 2 is applied to the problem (14), the following estimation can be written

$$
\|z\|_{C\left(\bar{\omega}_{N}\right)} \leq \propto^{-1}\|R\|_{C\left(\bar{\omega}_{N}\right)}
$$

For $R_{i}$, under the condition $A(x) \in \mathrm{C}^{1}[0, l]$

$$
\left|R_{i}\right| \leq C h_{i}, i=1,2, \ldots, N-1 \text {. }
$$

Hence,

$$
\begin{gathered}
\left|R_{i}\right| \leq a_{i} \hbar_{i}^{-1} \int_{x_{i-1}}^{x_{i+1}} d x \varphi_{i} \int_{x_{i-1}}^{x_{i+1}}\left|\frac{d u(\xi)}{d x}\right| T_{0}(x-\xi) d \xi \\
+\hbar_{i}^{-1} \int_{x_{i-1}}^{x_{i+1}}\left|a(x)-a\left(x_{i}\right)\right| \cdot|u(x)| \varphi_{i} d x \\
+\hbar_{i}^{-1}\left\{\int_{x_{i-1}}^{x_{i+1}}\left|f(x, u)-f\left(x_{i}, u\right)\right| \varphi_{i} d x+\int_{x_{i-1}}^{x_{i+1}}\left|f\left(x_{i}, u\right)-f\left(x_{i}, u\left(x_{i}\right)\right)\right| \varphi_{i} d x\right\} .
\end{gathered}
$$

To show (16), it is enough to show that the convergence rate of the following expression is $O\left(h_{i}\right)$

$$
\hbar_{i}^{-1} \int_{x_{i-1}}^{x_{i+1}}\left|f\left(x_{i}, u\right)-f\left(x_{i}, u\left(x_{i}\right)\right)\right| \varphi_{i} d x
$$

By using the mean value theorem, the condition $\left|\frac{\partial f\left(x_{i}, \widetilde{u}\right)}{\partial u}\right| \leq C$ and

$$
\left|u^{\prime}(x)\right| \leq C\left\{1+\frac{1}{\varepsilon}\left(e^{\frac{-\sqrt{\alpha} x}{\varepsilon}}+e^{\frac{-\sqrt{\alpha}(l-x)}{\varepsilon}}\right)\right\},
$$


we can write

$$
\begin{gathered}
\hbar_{i}^{-1} \int_{x_{i-1}}^{x_{i+1}}\left|f\left(x_{i}, u\right)-f\left(x_{i}, u\left(x_{i}\right)\right)\right| \varphi_{i} d x=\hbar_{i}^{-1} \int_{x_{i-1}}^{x_{i+1}}\left|\frac{\partial f\left(x_{i}, \tilde{u}\right)}{\partial u}\right| u(x)-u\left(x_{i}\right) \mid \varphi_{i} d x \\
\leq C \hbar_{i}^{-1} \int_{x_{i-1}}^{x_{i+1}} d x \varphi_{i} \int_{x_{i-1}}^{x_{i+1}}\left|\frac{d u(\xi)}{d x}\right| d \xi \\
\leq C \int_{x_{i-1}}^{x_{i}}\left\{1+\frac{1}{\varepsilon}\left(e^{\frac{-\sqrt{\alpha} \xi}{\varepsilon}}+e^{\left.\frac{-\sqrt{\alpha}(l-\xi)}{\varepsilon}\right)}\right)\right\} d \xi \\
\leq C\left\{h_{i}+(\sqrt{\alpha})^{-1}\left[e^{\frac{-\sqrt{\alpha} x_{i-1}}{\varepsilon}}-e^{\frac{-\sqrt{\alpha} x_{i}}{\varepsilon}}-e^{\frac{-\sqrt{\alpha}\left(l-x_{i}\right)}{\varepsilon}}+e^{\left.\left.\frac{-\sqrt{\alpha}\left(l-x_{i-1}\right)}{\varepsilon}\right]\right\}}\right.\right.
\end{gathered}
$$

We estimate separately $R_{i}$ in $\left[0, \sigma_{1}\right],\left[\sigma_{1}, \sigma_{2}\right]$ and $\left[\sigma_{2}, l\right]$. First, in $\left[0, \sigma_{1}\right]$, for $\sigma_{1}<\frac{1}{4}$

$$
\begin{gathered}
h_{i}=x_{i}-x_{i-1}=\alpha^{-1} \varepsilon\left[-\ln \left(1-\left(1-e^{-\frac{\alpha l}{4 \varepsilon}}\right) \frac{4 i}{N}\right)+\ln \left(1-\left(1-e^{-\frac{\alpha l}{4 \varepsilon}}\right) \frac{4(i-1)}{N}\right)\right], \\
\leq 4 \alpha^{-1}(1-\varepsilon) N^{-1}
\end{gathered}
$$

Besides $e^{\frac{-\sqrt{\alpha} x_{i-1}}{\varepsilon}}-e^{\frac{-\sqrt{\alpha} x_{i}}{\varepsilon}} \leq 4 \alpha^{-1}(1-\varepsilon) N^{-1}$ is obtained. Similarly, $e^{\frac{-\sqrt{\alpha}\left(l-x_{i-1}\right)}{\varepsilon}}-$ $e^{\frac{-\sqrt{\alpha}\left(l-x_{i}\right)}{\varepsilon}} \leq 4 \alpha^{-1}(1-\varepsilon) N^{-1}$ is found. If $\sigma_{1}=\frac{1}{4}$,

$$
\begin{aligned}
h_{i}=x_{i}-x_{i-1}= & \alpha^{-1} \varepsilon\left[-\ln \left(1-\left(1-e^{-\frac{\alpha l}{4 \varepsilon}}\right) \frac{4 i}{N}\right)+\ln \left(1-\left(1-e^{-\frac{\alpha l}{4 \varepsilon}}\right) \frac{4(i-1)}{N}\right)\right], \\
& \leq \alpha^{-1}\left(1-e^{-\frac{\alpha l}{4 \varepsilon}}\right) N^{-1} \leq \alpha^{-1} \varepsilon \frac{\alpha l}{4 \varepsilon} 4 N^{-1}=l N^{-1},
\end{aligned}
$$

Author's corrections 21.10.2022:

is found. Thus it is proven $\left|R_{i}\right| \leq C N^{-1}$ in $\left[0, \sigma_{1}\right]$. For $\left[\sigma_{1}, \sigma_{2}\right]$, it is written

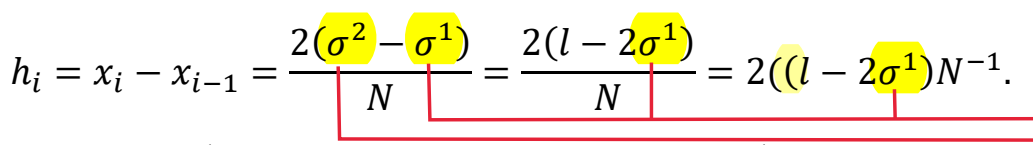

$h_{i} \leq l N^{-1}$ for $\sigma_{1}<\frac{1}{4} . h_{i}=l N^{-1}$ is obtained for $\sigma_{1}=\frac{1}{4}$. Therefore it is proven that $\left|R_{i}\right| \leq C N^{-1}$. Similarly, it is shown that $\left|R_{i}\right| \leq C N^{-1}$ in $\left[\sigma_{2}, l\right]$. The proof of the theorem is completed.

We now consider the necessary conditions that the convergence rate is $O\left(h^{2}\right)$. We express that with a theorem.

Theorem 2. If $a(x) \in C^{2}[0, l], f(x, u) \in C^{2}(D)$ and

$$
a^{\prime}(0)=a^{\prime}(l)=0,
$$


the convergence rate of difference scheme (12)-(13) is $O\left(h^{2}\right)$ and we can write as follows:

$$
\|y-u\|_{C\left(\omega_{N}\right)} \leq C h^{2} .
$$

Proof. The proof of the theorem is done in a similar manner [22].

\section{Results and discussion}

In this section, we present the numerical outcomes for the difference scheme

Author's corrections 21.10.2022: (12)-(13). Because of nonlinear terms, we suggest the Newton-Raphson technique for solving discretization. Applying this method, we get

$$
\begin{aligned}
& -\varepsilon^{2} \hbar_{i}^{-1}\left(y_{x, i}{ }^{(n)}-y_{\bar{x}, i}{ }^{(n)}\right) \\
& \frac{-\varepsilon^{2} h^{-2} \theta_{i}\left(y_{i+1}{ }^{(n)}-2 y_{i}^{(n)}+y_{i-1}^{(n)}\right)+a_{i} y_{i}^{(n)}-y_{i}^{(n)} \frac{\partial f\left(x_{i}, y_{i}^{(n-1)}\right)}{\partial u}}{=f\left(x_{i}, y_{i}^{(n-1)}\right)-y_{i}^{(n-1)} \frac{\partial f\left(x_{i}, y_{i}^{(n-1)}\right)}{\partial u}}
\end{aligned}
$$

Then, the difference problem (9)-(10) is modified accorrding to the following form

$$
\begin{gathered}
A_{i} y_{i-1}-C_{i} y_{i}+B_{i} y_{i+1}=-F_{i}, i=1, \ldots, N-1, \\
y_{0}=y_{N}=0,
\end{gathered}
$$

If the elimination method is considered, we obtain [25]

$$
\begin{aligned}
& \varepsilon^{2} \hbar_{i}^{-1} h_{i}^{-1} \\
& A_{i}=\varepsilon^{2} h_{i}^{-1} h_{i-1}^{-1}, B_{i}=\varepsilon^{2} h_{i}^{-1} h_{i+1}^{-1}, \\
& \varepsilon^{2} \hbar_{i}^{-1} h_{i+1}^{-1} \\
& \varepsilon^{2} \hbar_{i}^{-1}\left(h_{i}^{-1}+h_{i+1}^{-1}\right) \\
& C_{i}=2 \varepsilon^{2} \theta_{i} \theta_{i}+a_{i}-\frac{\partial f\left(x_{i}, y_{i}^{(n-1)}\right)}{\partial u} \\
& F_{i}=f\left(x_{i}, y_{i}^{(n-1)}\right)-y_{i}^{(n-1)} \frac{\partial f\left(x_{i}, y_{i}^{(n-1)}\right)}{\partial u},
\end{aligned}
$$

where the initial process is taken as $y_{i}^{(0)}=-2, i=1, \ldots, N-1$. Furthermore, the error estimates are denoted by

$$
e^{N}=\max _{i}\left|y_{i}{ }^{N}-u_{i}^{2 N}\right|,
$$

and the convergence rates are calculated as follows

$$
p_{u}{ }^{N}=\frac{\ln \left(e^{N} / e^{2 N}\right)}{\ln 2} .
$$

Example 1. We consider following the singularly perturbed nonlinear reaction-diffusion equation with boundary conditions 


$$
\begin{gathered}
-\varepsilon^{2} u^{\prime \prime}(x)+x(1-x) u(x)=u+u^{2} ; x \in(0,1), \\
u(0)=u(1)=0 .
\end{gathered}
$$

The computational results are presented in Table 1 for different values of $\varepsilon$ and $N$.

Table 1. Error approximations and convergence rates for Example 1

\begin{tabular}{|c|c|c|c|c|c|}
\hline \multicolumn{2}{|c|}{$\varepsilon$} & $N=32$ & $N=64$ & $N=128$ & $N=256$ \\
\hline \multirow{3}{*}{$10^{-2}$} & $e^{N}$ & 0.0799526742 & 0.014754788 & 0.0035099236 & 0.0008603976 \\
\cline { 2 - 6 } & $e^{2 N}$ & 0.0147547885 & 0.0034043440 & 0.0008603976 & 0.0002140310 \\
\cline { 2 - 6 } & $p$ & 2.4379630428 & 2.1157345182 & 2.0283641948 & 2.0071840245 \\
\hline \multirow{3}{*}{$10^{-4}$} & $e^{N}$ & 0.0828403312 & 0.0151691846 & 0.0035920895 & 0.0008799596 \\
\cline { 2 - 6 } & $e^{2 N}$ & 0.0151691846 & 0.0034750245 & 0.0008799596 & 0.0002188600 \\
\cline { 2 - 6 } $10^{-6}$ & $p$ & 2.4491897825 & 2.1260484965 & 2.0293141627 & 2.0074289004 \\
\hline \multirow{4}{*}{$10^{-8}$} & $e^{N}$ & 0.0828699525 & 0.0151733736 & 0.0035929183 & 0.0008801565 \\
\cline { 2 - 6 } & $e^{2 N}$ & 0.0151733736 & 0.0034757402 & 0.0008801565 & 0.0002189086 \\
\cline { 2 - 6 } & $p$ & 2.4493072074 & 2.1261497204 & 2.0293240973 & 2.0074314626 \\
\hline \multirow{3}{*}{$10^{-10}$} & $e^{N}$ & 0.0828702685 & 0.0151734155 & 0.0035929266 & 0.0008801585 \\
\cline { 2 - 6 } & $e^{2 N}$ & 0.0151734155 & 0.0034757474 & 0.0008801585 & 0.0002189091 \\
\hline & $p$ & 2.4493087244 & 2.1261507342 & 2.0293241968 & 2.0074314878 \\
\cline { 2 - 6 } & $e^{N}$ & 0.0828706110 & 0.0151735008 & 0.0035929284 & 0.0008801633 \\
\hline \multirow{3}{*}{$10^{-12}$} & 0.0151735008 & 0.0034757482 & 0.0008801633 & 0.0002189097 \\
\cline { 2 - 6 } & $p$ & 2.4493065792 & 2.1261585109 & 2.0293170202 & 2.0074355070 \\
\cline { 2 - 6 } & $e^{2 N}$ & 0.0828702899 & 0.0151734160 & 0.0035940285 & 0.0008804717 \\
\cline { 2 - 6 } & 0.0151734160 & 0.0034765455 & 0.0008804717 & 0.0002189558 \\
\hline
\end{tabular}

Also, it is observed that the numerical solution of Example 1 has two boundary layers from Figure 1.

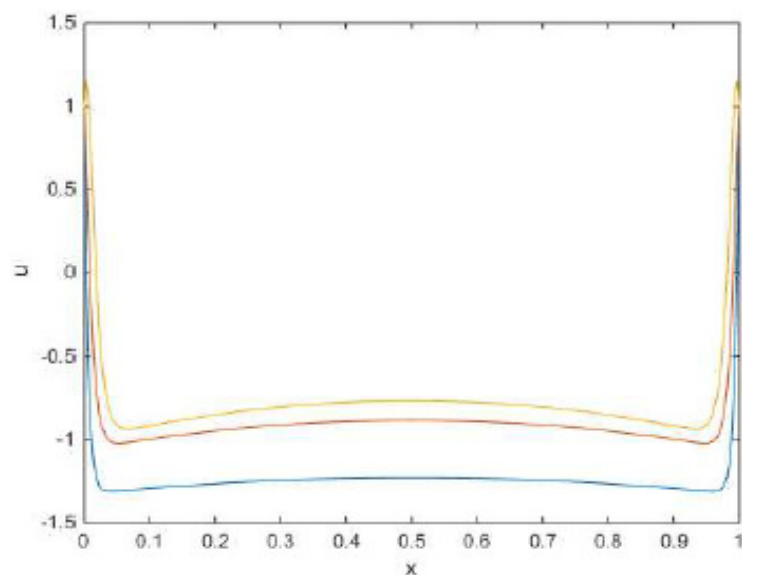

Fig. 1. Numerical solution for $\varepsilon=10^{-4}$ and $N=64$ 
Example 2. We take into account another singularly perturbed boundary value problem

$$
\begin{gathered}
-\varepsilon^{2} u^{\prime \prime}(x)+e^{x} u(x)=\sin u+u ; x \in(0,1), \\
u(0)=u(1)=0 .
\end{gathered}
$$

The numerical outcomes are demonstrated in Table 2.

Table 2. Error approximations and convergence rates for Example 2

\begin{tabular}{|c|c|c|c|c|c|}
\hline \multicolumn{2}{|c|}{$\varepsilon$} & $N=32$ & $N=64$ & $N=128$ & $N=256$ \\
\hline \multirow{3}{*}{$10^{-2}$} & $e^{N}$ & 0.0560907495 & 0.0131986907 & 0.0032395318 & 0.0002013206 \\
\cline { 2 - 6 } & $e^{2 N}$ & 0.0131986907 & 0.0032395318 & 0.0008061984 & 0.0002013206 \\
\cline { 2 - 6 } & $p$ & 2.0873680382 & 2.0265376068 & 2.0065784439 & 2.0016403685 \\
\hline \multirow{3}{*}{$10^{-4}$} & $e^{N}$ & 0.0567253715 & 0.0133976816 & 0.0032893099 & 0.0008230667 \\
\cline { 2 - 6 } & $e^{2 N}$ & 0.0133976816 & 0.0032893099 & 0.0008186327 & 0.0002055001 \\
\cline { 2 - 6 } & $p$ & 2.0820107768 & 2.0261265381 & 2.0064966745 & 2.0018704650 \\
\hline \multirow{3}{*}{$10^{-6}$} & $e^{N}$ & 0.0567533914 & 0.0134026241 & 0.0032904792 & 0.0008234119 \\
\cline { 2 - 6 } & $e^{2 N}$ & 0.0134026241 & 0.0032904792 & 0.0008189231 & 0.0002055862 \\
\cline { 2 - 6 } & $p$ & 2.0821911117 & 2.0261458699 & 2.0064978773 & 2.0018706756 \\
\hline \multirow{3}{*}{$10^{-10}$} & $e^{N}$ & 0.0567571501 & 0.0134027487 & 0.0032904927 & 0.0008234155 \\
\cline { 2 - 6 } & $e^{2 N}$ & 0.0134027487 & 0.0032904927 & 0.0008189261 & 0.0002055871 \\
\cline { 2 - 6 } & $p$ & 2.0822732420 & 2.0261533604 & 2.0064984282 & 2.0018707102 \\
\cline { 2 - 6 } & $e^{N}$ & 0.0567592200 & 0.0134027915 & 0.0032904936 & 0.0008234155 \\
\cline { 2 - 6 } $10^{-12}$ & 0.0134027915 & 0.0032904936 & 0.0008189262 & 0.0002055871 \\
\cline { 2 - 6 } & $e^{N}$ & 2.0823212534 & 2.0261575862 & 2.0064987317 & 2.0018707282 \\
\cline { 2 - 6 } & $e^{2 N}$ & 0.0567605773 & 0.0134028187 & 0.0032904941 & 0.0008234155 \\
\hline
\end{tabular}

Figure 2 shows behavior of the numerical solution for Example 2.

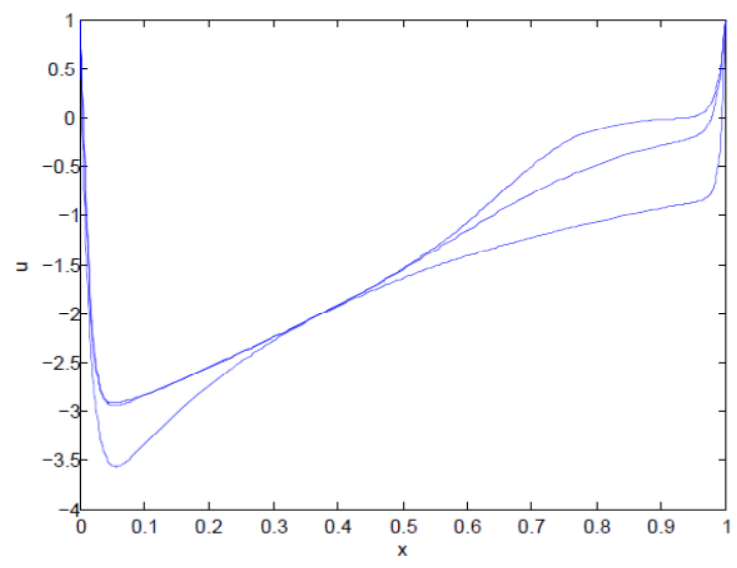

Fig. 2. Numerical solution for $\varepsilon=10^{-6}$ and $N=256$ 
The exact solutions of the Examples 1-2 are unknown. To obtain the experimental rates of convergence, we compare the computed solution with the solution on a mesh that is twice as fine. The proposed scheme is useful and simple to solve such problems. Moreover, it is uniform and yields more accurate results than some existing ones presented in the literature. Furthermore, the presented scheme can be implemented by a combination with other techniques for higher dimensional forms of these problems.

\section{Conclusions}

In this article, the classical difference scheme was constructed on adaptive mesh for nonlinear reaction-diffusion equations with a perturbation parameter. By using asymptotic evaluations, the convergence analysis of the method was successfully investigated. In the algorithm, the Newton-Raphson method was used because of nonlinear terms. The theoretical outcomes were tested on samples. The computational results were illustrated in tables. Based on the numerical experiments, we observe that the orders of convergence are close to 2. Numerical research can be carried out for different types such as delay, parabolic, elliptic, higher dimensional, etc.

\section{References}

[1] Chakravarthy, P.P., \& Kumar, K. (2017). A novel method for singularly perturbed delay differential equations of reaction-diffusion type. Differential Equations and Dynamical Systems. https://doi.org/10.1007/s12591-017-0399-x.

[2] Zeng, F., Lu, F., Li, C., Burrage, K., Tuner, I., \& Anh, V. (2014). A Crank-Nicolson ADI spectral method for a two-dimensional Riesz space fractional nonlinear reaction-diffusion equation. SIAM Journal on Numerical Analysis, 52(6), 2599-2622.

[3] Mitchell, A.R., \& Bruch, Jr. J.C. (1985). A numerical study of chaos in a reaction-diffusion equation. Numerical Methods for Partial Differential Equations, 1(1), 13-23.

[4] Baeumer, B., Kovacs, M., \& Meerschaert, M.M. (2008). Numerical solutions for fractional reaction-diffusion equations. Computer and Mathematics with Applications, 55, 2212-2226.

[5] Das, S., Gupta, P.K., \& Ghosh, P. (2011). An approximate solution of nonlinear fractional reaction-diffusion equation. Applied Mathematical Modelling, 35, 4071-4076.

[6] Pulkkinen, A. (2011). Blow-up profiles of solutions for the exponential reaction-diffusion equations. Mathematical Methods in the Applied Sciences. https://doi.org/10.1002/mma.1501.

[7] Liao, W., \& Yan, Y. (2010). Singly diagonally 1mplicit Runge-Kutta method for time-dependent reaction-diffusion equation. Numerical Methods for Partial Differential Equations, 27(6), 1423-1441.

[8] Kopteva, N., \& Stynes, M. (2011). Stabilised approximation of interior-layer solutions of a singularly perturbed semilinear reaction-diffusion problem. Numerische Mathematik, 119(4), 787-810.

[9] Cheng, R. (2006). Singularly perturbed problem for non-local reaction-diffusion equations involving two small parameters. Journal of Shanghai University (English Edition), 10(6), 479-483. 
[10] Zhu, G., \& Chen, S. (2010). Convergence and superconvergence analysis of an anisotropic noncoforming finite element methods for singularly perturbed reaction-diffusion problems. Computational and Applied Mathematics, 234(10), 3048-3063.

[11] Shishkin, G.I., \& Shishkina, L.P. (2011). Improved approximations of the solution and derivatives to a singularly perturbed reaction-diffusion equation based on the solution deceomposition method. Computational Mathematics and Mathematical Physics, 51(6), 1020-1049.

[12] Siraj, M.K., Duressa, G.F., \& Bullo, T.A. (2019). Fourth-order stable central difference with Richardson extrapolation method for second-order self-adjoint singularly perturbed boundary value problems. Journal of Egyptian Mathematical Society, 27, 50.

[13] Gelu, F.W., Duressa, G.F., \& Bullo, T.A. (2017). Sixth-order compact finite difference method for singularly perturbed 1D reaction-diffusion problems. Journal of Taibah University for Science, 11, 302-308.

[14] Clavero, C., \& Gracia, J.L. (2012). A high order HODIE finite difference scheme for 1D parabolic singularly perturbed reaction-diffusion problems. Applied Mathematics and Computation, 218(9), 5067-5080.

[15] Raza, N., \& Butt, A.R. (2013). Numerical solutions of singularly perturbed reaction diffusion equation with Sobolev gradients. Journal of Function Spaces. https://doi.org/10.1155/2013/ 542897.

[16] Srivastava, A. (2017). Numerical simulation of singularly perturbed reaction-diffusion equations using finite element method. Computational Mathematics and Modelling, 28, 3.

[17] Cen, Z., Le, A., \& Xu, A. (2017). A high-order finite difference scheme for a singularly perturbed reaction-diffusion problem with an interior layer. Advences in Difference Equations, 202.

[18] Hong, Y., Jung, C., \& Laminie, J. (2013). Singularly perturbed reaction-diffusion equations in a circle with numerical applications. International Journal of Computer Mathematics, 90, $11,2308-2325$.

[19] He, X., \& Wang, K. (2019). Uniformly convergent novel finite difference methods for singularly perturbed reaction-diffusion equations. Numerical Methods for Partial Differential Equations. https://doi.org/10.1002/num.22405.

[20] Singh, J., Kumar, S., \& Kumar, M. (2018). A domain decomposition method for solving singularly perturbed parabolic reaction-diffusion problesm with time delay. Numerical Methods for Partial Differential Equation, 34(5), 1849-1866.

[21] Sekar, E., \& Tamilselvan, A. (2019). Parameter uniform method for a singularly perturbed system of delay differential equations of reaction-diffusion type wtih integral boundary conditions. International Journal of Applied and Computational Mathematics, 5, 85.

[22] Amiraliyev, G.M., \& Duru, H. (2002). Nümerik Analiz. Pegem Yayıncılık.

[23] Boglaev, I.P. (1984). Approximate solution of a nonlinear boundary value problem with a small parameter for the highest-order differential. USSR Computational Mathematics and Mathematical Physics, 24(6), 30-35.

[24] Amiraliyev, G.M., \& Mamedov, Y.D. (1995). Difference schemes on the uniform mesh for singularly perturbed pseudo-parabolic equations. Turkish Journal of Mathematics, 19, 207-222.

[25] Samarskii, A.A. (2001). The Theory of Difference Schemes. Moscow; M.V. Lomonosov State University. 